\title{
Identification of a missense variant in the WFS1 gene that causes a mild form of Wolfram syndrome and is associated with risk for type 2 diabetes in Ashkenazi Jewish individuals
}

\author{
Vikas Bansal $^{1} \cdot$ Bernhard O. Boehm $^{2,3,4} \cdot$ Ariel Darvasi $^{5}$
}

Received: 21 March 2018 / Accepted: 15 June 2018 / Published online: 16 July 2018

(C) Springer-Verlag GmbH Germany, part of Springer Nature 2018

\begin{abstract}
Aims/hypothesis Wolfram syndrome is a rare, autosomal recessive syndrome characterised by juvenile-onset diabetes and optic atrophy and is caused by bi-allelic mutations in the WFS1 gene. In a recent sequencing study, an individual with juvenile-onset diabetes was observed to be homozygous for a rare missense variant (c.1672C>T, p.R558C) in the WFS1 gene. The aim of this study was to perform the genetic characterisation of this variant and to determine whether it is causal for young-onset diabetes and Wolfram syndrome.

Methods We analysed the allele frequency of the missense variant in multiple variant databases. We genotyped the variant in 475 individuals with type 1 diabetes and 2237 control individuals of Ashkenazi Jewish ancestry and analysed the phenotypes of homozygotes. We also investigated the association of this variant with risk for type 2 diabetes using genotype and sequence data for type 2 diabetes cases and controls.

Results The missense variant demonstrated an allele frequency of $1.4 \%$ in individuals of Ashkenazi Jewish ancestry, 60-fold higher than in other populations. Genotyping of this variant in 475 individuals diagnosed with type 1 diabetes identified eight homozygotes compared with none in 2237 control individuals (genotype relative risk $135.3, p=3.4 \times 10^{-15}$ ). The age at diagnosis of diabetes for these eight individuals (17.8 \pm 8.3 years) was several times greater than for typical Wolfram syndrome ( $5 \pm 4$ years). Further, optic atrophy was observed in only one of the eight individuals, while another individual had the Wolfram syndrome-relevant phenotype of neurogenic bladder. Analysis of sequence and genotype data in two case-control cohorts of Ashkenazi ancestry demonstrated that this variant is also associated with an increased risk of type 2 diabetes in heterozygotes (OR 1.81, $p=0.004$ ).

Conclusions/interpretation We have identified a low-frequency coding variant in the WFS1 gene that is enriched in Ashkenazi Jewish individuals and causes a mild form of Wolfram syndrome characterised by young-onset diabetes and reduced penetrance for optic atrophy. This variant should be considered for genetic testing in individuals of Ashkenazi ancestry diagnosed with young-onset non-autoimmune diabetes and should be included in Ashkenazi carrier screening panels.
\end{abstract}

Keywords Ashkenazi Jewish · Carrier screening · Genetic testing · Juvenile-onset diabetes · Optic atrophy · Type 1 diabetes · Type 2 diabetes $\cdot$ Wolfram syndrome

Electronic supplementary material The online version of this article (https://doi.org/10.1007/s00125-018-4690-3) contains peer-reviewed but unedited supplementary material, which is available to authorised users.

Vikas Bansal

vibansal@ucsd.edu

1 Department of Pediatrics, University of California San Diego, 9500 Gilman Drive, La Jolla, CA 92093, USA

2 Department of Internal Medicine I, Ulm University Medical Centre, Ulm, Germany
3 Lee Kong Chian School of Medicine, Nanyang Technological University, Singapore, Singapore

4 Imperial College London, London, UK

5 Department of Genetics, The Institute of Life Sciences, The Hebrew University of Jerusalem, Givat Ram, Jerusalem, Israel 


\section{Research in context}

\section{What is already known about this subject?}

- Wolfram syndrome is a rare autosomal recessive disorder characterised by juvenile-onset diabetes and optic atrophy

- In the absence of optic atrophy, Wolfram syndrome can be clinically misdiagnosed as non-autoimmune type 1 diabetes

- A rare missense variant (c.1672C>T, p.R558C) in the WFS1 gene has previously been observed in homozygous state in two individuals from different studies, one with an atypical form of Wolfram syndrome and another with juvenileonset diabetes without optic atrophy

\section{What is the key question?}

- Does a low-frequency missense variant in the WFS1 gene cause recessive young-onset non-autoimmune diabetes and other phenotypes observed in Wolfram syndrome?

\section{What are the new findings?}

- A missense variant, p.R558C, in the WFS1 gene is enriched in the Ashkenazi Jewish population (allele frequency 1.4\%) and causes a mild form of Wolfram syndrome characterised by young-onset diabetes (mean age at diagnosis 17.8 years) and incomplete penetrance for optic atrophy

- Individuals with this variant are frequently misdiagnosed as having type 1 diabetes

- The same variant is associated with an increased risk of type 2 diabetes in heterozygous carriers

How might this impact on clinical practice in the foreseeable future?

- Genetic testing for this variant in individuals diagnosed with early-onset diabetes who are negative for islet antibodies can identify individuals with undiagnosed Wolfram syndrome, even in the absence of optic atrophy

\section{Abbreviations \\ GAD65 $65 \mathrm{kDa}$ isoform of GAD \\ HUGR Hebrew University Genetic Resource}

\section{Introduction}

Wolfram syndrome (Mendelian Inheritance in Man [MIM] no. 222300) is a rare, autosomal recessive neurodegenerative disorder characterised by juvenile-onset diabetes and optic atrophy [1]. A range of phenotypes, including diabetes insipidus, renal-tract abnormalities and sensorineural deafness, are observed in individuals with Wolfram syndrome [2]. Insulin-deficient, islet antibody-negative diabetes is usually the first phenotype observed in affected individuals (median age of 6 years), while optic atrophy occurs later in life (median age of 11 years). Sensorineural deafness (median age of 20 years) and neurological abnormalities (median age of 30 years) occur in a significant fraction of affected individuals. Wolfram syndrome is mainly caused by bi-allelic mutations in the WFS1 gene. This gene encodes a transmembrane protein (wolframin), which has an important role in regulating calcium levels in the endoplasmic reticulum [3].

Heterozygous mutations in WFS1 have been reported to be associated with less severe phenotypes than those observed in Wolfram syndrome, including low-frequency sensorineural hearing loss without diabetes [4, 5], autosomal dominant diabetes [6], optic atrophy [7], hearing loss [7] and congenital cataracts [8]. A recent study [9] identified dominant missense mutations in WFS1 in multiple individuals with diabetes diagnosed in the first year of life, sensorineural deafness and congenital cataracts. Therefore, the genotype-phenotype relationship for mutations in WFS1 is complex, with both recessive and autosomal dominant modes of inheritance and overlapping phenotypes.

Since the discovery of the WFS1 gene in 1998, more than 300 different mutations have been identified in this gene in individuals diagnosed with Wolfram syndrome [10, 11]. Many individuals with Wolfram syndrome, particularly those with consanguinity, are homozygous for loss-of-function mutations while others are compound heterozygous for deleterious mutations. Homozygous missense mutations in WFS1 are less frequently observed in individuals with Wolfram syndrome when compared with loss-of-function variants. Cano et al [10] reported that the clinical expression of Wolfram syndrome is more complete in individuals with truncating mutations in WFS1. Analysis of the genotype-phenotype relationship in collections of individuals diagnosed with Wolfram syndrome has shown that the age at diagnosis of both diabetes and optic atrophy is significantly greater in individuals with two missense mutations than in those with a single missense mutation or no missense mutation $[11,12]$. 
Many individuals with Wolfram syndrome are misdiagnosed as having type 1 diabetes in the absence of optic atrophy [13,14]. Identification of Wolfram syndrome in individuals diagnosed with diabetes in childhood or adolescence is important since the management and prognosis of Wolfram syndrome is different to that of type 1 diabetes. In a recent targeted sequencing study of more than 6800 individuals from Germany [15], one individual with juvenile-onset diabetes was observed to be homozygous for a rare missense variant (NM_001145853:c.1672C $>$ T, p.R558C) in the WFS1 gene. However, this individual did not have optic atrophy or other phenotypes frequently observed in Wolfram syndrome. Notably, another individual with an atypical presentation of Wolfram syndrome (diabetes diagnosed at 33 years of age and optic atrophy at 53 years) was previously reported to be homozygous for the same variant [16]. This variant has also been observed in combination with other variants in multiple individuals with Wolfram syndrome [10]. In this study, we investigated this rare variant further to determine whether it is causal for young-onset diabetes and Wolfram syndrome in homozygous individuals. Using genotype data for Ashkenazi Jewish individuals, we tested this variant for association with type 1 diabetes and type 2 diabetes in homozygous and heterozygous carriers, respectively.

\section{Methods}

Ashkenazi Jewish samples Four hundred and seventy-five individuals of Ashkenazi Jewish ancestry with type 1 diabetes were obtained from the Hebrew University Genetic Resource (HUGR). The inclusion criteria specified that participants were diagnosed with type 1 diabetes according to the aetiological classification of diabetes mellitus proposed by the International Expert Committee under the sponsorship of the American Diabetes Association in May 1997 and that all four grandparents of each participant were self-reported to be of Ashkenazi Jewish ethnic origin. Thirty-eight per cent of the individuals were positive for islet-cell autoantibodies against the $65 \mathrm{kDa}$ isoform of GAD (GAD65), islet-cell antigen 512 or insulin, and were likely to have autoimmune type 1 diabetes [17]. The remaining individuals were categorised as having idiopathic diabetes or an unknown form of diabetes. The age at diabetes diagnosis for the 475 individuals was $18.6 \pm$ 13.6 years (ESM Table 1), at which point $42 \%$ had ketoacidosis.

A further 1131 individuals with type 2 diabetes and 2237 control individuals were obtained from HUGR (see ESM Table 1). The individuals with type 2 diabetes were collected in Israel and all individuals self-reported that all four grandparents were Ashkenazi Jews [18]. The mean age at diagnosis in the 1131 individuals with type 2 diabetes was 53 years. The control individuals reported no chronic disease and were not screened for diabetes (mean age 48.9 years). For association with type 2 diabetes, a second set of Ashkenazi Jewish samples (506 individuals with diabetes and 352 control individuals without) from the T2D-GENES exome sequencing project [19] was used and genotype data was obtained from dbGaP.

Genotyping Genotyping of the p.R558C (rs199946797) and p.R611H (rs734312) variants in the Ashkenazi Jewish samples from the HUGR cohort was performed by LGC Genomics (Middlesex, UK) using the KASP assay [20]. Twenty-two individuals with type 2 diabetes and 57 control individuals had missing genotypes at all genotyped variants (p.R558C, p.R611H and three additional variants) and were excluded from the analysis. For the remaining samples, the genotyping success rate was $>97.5 \%$.

Statistical analysis Case-control association analysis for type 2 diabetes was done using Fisher's exact test and logistic regression in Matlab. An inverse variance-based approach [21] (with fixed effect size) was used to combine the association statistics for the two Ashkenazi Jewish type 2 diabetes case-control datasets. Individuals with missing genotypes were excluded from the association analysis. The recessive allele frequency based test (RAFT; https://personal.broadinstitute.org/rigel/raft; accessed 1 November 2017) [22] was used to perform the association analysis under a recessive model for type 1 diabetes. Hardy-Weinberg equilibrium $p$ values were calculated using a $1 d f \chi^{2}$ test. For the T2D-GENES data, we used an online burden test from the Type 2 Diabetes Knowledge Portal (www.type2diabetesgenetics.org; accessed 1 November 2017) to test the association of heterozygous loss-of-function variants with type 2 diabetes. For the targeted sequence data from Bansal et al [15], we compared the counts of the number of carriers of loss-of-function variants using Fisher's exact test.

Haplotype and sequence analysis Haplotype phasing of the genotypes at the WFS1 gene for the Ashkenazi Jewish individuals from the T2D-GENES dataset was done using the PHASE program [23] (v2.1). Analysis of sequence reads to determine the phase between the p.R558C variant and neighbouring heterozygous variants was performed using the HapCUT software (v0.7; https://github.com/vibansal/hapcut; accessed 1 November 2017) [24] . Multiple sequence alignment for the WFS1 protein sequence across species was obtained from the NCBI HomoloGene server (https://www. ncbi.nlm.nih.gov/homologene; accessed 20 January 2018).

Ethics approval For the HUGR samples, all individuals or their legal representative signed an informed consent form and the entire sample collection was approved by the relevant Israeli national ethics committee. All individuals from the Ulm diabetes cohort gave informed consent for use of their DNA samples for genetic studies [15]. The study was carried 
out in accordance with the principles of the Declaration of Helsinki.

\section{Results}

In a recent sequencing study of monogenic diabetes genes in 6888 individuals from Germany [15], a single individual, diagnosed with diabetes at 14 years of age, was reported to be homozygous for a missense variant (NM_001145853:c.1672C >T, p.R558C, rs199946797) in the WFS1 gene. To determine whether this individual had phenotypes consistent with Wolfram syndrome, we obtained additional phenotype information. The individual initially presented with a high fasting glucose level ( $>10 \mathrm{mmol} / \mathrm{l}$ ) without ketosis but required treatment with insulin and also had ketoacidosis after 6 months. No circulating islet-cell antibodies or antibodies against the islet-cell antigens GAD65, islet-cell antigen 2 (IA2) and zinc transporter 8 (ZnT8) were detected. Ultrasound examination of the individual revealed a right-sided pyeloectasia (i.e. distension of pelvis of kidney). The individual's mother had non-insulin-dependent diabetes mellitus considered as type 2 diabetes. Although the individual did not have optic atrophy or other Wolfram syndrome-relevant phenotypes, the phenotype of insulin-dependent, juvenile-onset non-autoimmune diabetes was consistent with the diabetic phenotype observed in Wolfram syndrome.

\section{The p.R558C allele is enriched in the Ashkenazi Jewish popu-} lation The allele frequency of the p.R558C variant in a dataset of 6888 European individuals was observed to be $0.07 \%$ [15]. Homozygous genotypes at such low-frequency variants can be the result of parental relatedness or be due to an unknown founder mutation. Analysis of the targeted sequence data for this individual did not show any evidence of excess homozygosity. To explore the possibility that this variant is frequent in non-European populations, we performed a search in several exome sequencing-derived variant databases such as Exome Aggregation Consortium [25], NHLBI Exome Sequencing Project [26], 1000 Genomes Project and GnomAD (http:// gnomad.broadinstitute.org; accessed 1 November 2017) [25]. Using data from the GnomAD database, we found that the p.R558C variant has a minor allele frequency of $1.4 \%$ in the Ashkenazi Jewish population $(n=5075), 60$-fold greater than in non-Finnish Europeans and all other populations (ESM Table 2). This finding was consistent with the family history of the previously reported p.R558C homozygous individual [16] whose parents were Ashkenazi Jewish.

Eight Ashkenazi Jewish individuals with type 1 diabetes are p.R558C homozygotes To assess whether the p.R558C variant is associated with diabetes, we genotyped the variant in 475 Ashkenazi Jewish individuals from Israel diagnosed with type 1 diabetes and 2237 control individuals of the same ancestry (see Methods). Notably, $81 \%$ of these individuals were diagnosed with diabetes before the age of 30 years (age at diagnosis $18.6 \pm 13.6$ years) and $62 \%$ had no evidence of autoimmunity (negative for islet-cell autoantibodies). Genotyping revealed that eight individuals were homozygous and six were heterozygous for this variant (Table 1 ), a strong violation of Hardy-Weinberg equilibrium $\left(p=2.6 \times 10^{-53}, 1 d f \chi^{2}\right.$ test $)$. In contrast, among 2237 control individuals, no homozygotes and 49 heterozygotes were observed. We assessed the association of the p.R558C variant with diabetes under a recessive model using a recent statistical method [22]. This allele frequency-based test showed that the p.R558C variant is strongly associated with an increased risk of diabetes in homozygous individuals (genotype relative risk 135.3, $p=3.4 \times$ $\left.10^{-15}\right)$. To confirm the genotypes, we repeated the genotyping in the 475 individuals with type 1 diabetes. The genotyping was done using the same technology but using a different primer (on the strand opposite to that used in the initial genotyping). We observed a $99.8 \%$ genotype concordance rate and confirmed the genotype for each of the eight homozygous individuals.

All eight homozygous individuals were diagnosed with diabetes before the age of 30 years (mean age at diagnosis $17.8 \pm 8.3$ years, Table 2). The mean $\mathrm{HbA}_{1 \mathrm{c}}$ level of the eight individuals was $55 \mathrm{mmol} / \mathrm{mol}(7.2 \%)$, indicative of good glycaemic control. Only one of these eight individuals, diagnosed with diabetes at the age of 18 years, had the phenotype of optic atrophy (Table 2). In addition, one individual was diagnosed with keratoconus (thinning of the cornea) and another had the phenotype of neurogenic bladder, a phenotype that is sometimes observed in individuals with Wolfram syndrome [1,27]. Three individuals (excluding the one individual with optic atrophy) had the diabetic complication of retinopathy (Table 2). The diabetic retinopathy phenotype is likely to be related to diabetes since $22.2 \%$ of individuals in the cohort had this phenotype and diabetic retinopathy has not been associated with Wolfram syndrome. Three individuals also had ketoacidosis, a complication that is not frequently seen in individuals with Wolfram syndrome [28], while two individuals were positive for islet-cell autoantibodies (Table 2).

The age at diagnosis of diabetes $(17.8 \pm 8.3$ years $)$ was several times greater than that observed for Wolfram syndrome ( $5 \pm 4$ years) $[27,29]$. Individuals with Wolfram syndrome have a median lifespan of 30 years [29, 30]. In contrast, the mean age at recruitment of the eight homozygous individuals was 44.5 years (range 25-67 years). Only two of the eight homozygous individuals, one with optic atrophy (age 44 years) and another with neurogenic bladder (age 58 years), had additional Wolfram syndrome-relevant phenotypes. Overall, the phenotype data for the eight homozygous individuals identified in this study and the two previously reported homozygous individuals $[15,16]$ indicates that homozygosity for this missense variant leads to a mild or less severe form of 
Table 1 Genotype counts, allele frequencies and association statistics for the p.R558C variant (NM_001145853:c.1672C>T) in Ashkenazi Jewish samples

\begin{tabular}{|c|c|c|c|c|c|c|c|c|}
\hline \multirow[t]{2}{*}{ Cohort } & \multirow[t]{2}{*}{ Phenotype } & \multirow[t]{2}{*}{$n$} & \multicolumn{3}{|c|}{ Genotype counts } & \multirow[t]{2}{*}{ Allele frequency $(\%)$} & \multirow{2}{*}{$\begin{array}{l}\text { Measure of } \\
\text { association }\end{array}$} & \multirow[t]{2}{*}{$p$ value } \\
\hline & & & $\mathrm{C} / \mathrm{C}$ & $\mathrm{C} / \mathrm{T}$ & $\mathrm{T} / \mathrm{T}$ & & & \\
\hline HUGR & Type 1 diabetes & 475 & 441 & 6 & 8 & 2.42 & $135.3^{\mathrm{a}}$ & $3.4 \times 10^{-15}$ \\
\hline $\begin{array}{l}\text { HUGR } \\
\text { HUGR }\end{array}$ & $\begin{array}{l}\text { Type } 2 \text { diabetes } \\
\text { Control group }\end{array}$ & $\begin{array}{l}1131 \\
2237\end{array}$ & $\begin{array}{l}1041 \\
2081\end{array}$ & $\begin{array}{l}40 \\
49\end{array}$ & $\begin{array}{l}0 \\
0\end{array}$ & $\begin{array}{l}1.85 \\
1.15\end{array}$ & $1.63(1.07,2.49)^{\mathrm{b}}$ & 0.024 \\
\hline $\begin{array}{l}\text { T2D-GENES } \\
\text { T2D-GENES }\end{array}$ & $\begin{array}{l}\text { Type } 2 \text { diabetes } \\
\text { Control group }\end{array}$ & $\begin{array}{l}506 \\
352\end{array}$ & $\begin{array}{l}488 \\
349\end{array}$ & $\begin{array}{r}18 \\
3\end{array}$ & $\begin{array}{l}0 \\
0\end{array}$ & $\begin{array}{l}1.78 \\
0.43\end{array}$ & $4.29(1.25,14.68)^{\mathrm{b}}$ & 0.02 \\
\hline
\end{tabular}

The same set of control samples (HUGR) were used for the type 1 diabetes and type 2 diabetes association analysis

${ }^{\text {a }}$ Genotype relative risk under a recessive model

${ }^{\mathrm{b}} \mathrm{OR}(95 \% \mathrm{CI})$ for type 2 diabetes per minor allele under an additive model

Wolfram syndrome characterised by young-onset diabetes (age at diagnosis $<30$ years) and reduced penetrance for additional Wolfram syndrome-relevant phenotypes such as optic atrophy and neurogenic bladder.

\section{p.R558C is associated with increased risk for type 2 diabetes} in heterozygous carriers Next, we genotyped the p.R558C variant in Ashkenazi Jewish individuals with type 2 diabetes $(n=$ 1131) from Israel [18]. Although no homozygotes were identified, we observed an increased frequency of heterozygotes in the diabetic individuals compared with control individuals (Table 1, OR 1.63, $p=0.024$ ). The mean age at diagnosis for the heterozygous variant carriers was not significantly different compared with the overall group (51.7 years vs 53.0 years, $p=0.56$ using rank sum test). To further assess the association of the p.R558C variant with type 2 diabetes, we used wholeexome data for $\sim 17,000$ individuals from the T2D-GENES project [19]. This dataset includes 506 individuals with type 2 diabetes and 352 control individuals of Ashkenazi Jewish ancestry. The p.R558C variant was also associated with an increased risk of type 2 diabetes in this case-control dataset (OR 4.29, $p=0.02$, Table 1). Therefore, data from two independent Ashkenazi Jewish case-control datasets indicate that the p.R558C variant, in addition to being pathogenic for youngonset diabetes in homozygotes, is also associated with an increased risk for type 2 diabetes in heterozygous carriers (combined OR 1.81, $p=0.0038$ ). To test whether deleterious coding variants in the WFS1 gene are also associated with an increased risk of type 2 diabetes in heterozygotes, we performed burden tests using predicted loss-of-function (stop-gain, splice-site disrupting and frameshift) variants identified in two large case-control datasets for type 2 diabetes, the T2D-GENES project [19] and a recent targeted sequencing study [15]. However, we did not observe any association between the loss-offunction variants and risk for type 2 diabetes (ESM Table 3).

Common variants in the WFS1 gene have also been associated with increased risk for type 2 diabetes in candidate gene and genome-wide association studies [31-33]. The p.R558C

Table 2 Clinical phenotypes for eight p.R558C homozygotes of Ashkenazi Jewish ancestry with type 1 diabetes

\begin{tabular}{|c|c|c|c|c|c|c|c|}
\hline Individual ID & $\begin{array}{l}\text { Age at } \\
\text { diagnosis (years) }\end{array}$ & $\begin{array}{l}\text { Age at } \\
\text { recruitment } \\
\text { (years) }\end{array}$ & Ocular phenotype & Additional phenotypes & $\begin{array}{l}\mathrm{HbA}_{1 \mathrm{c}} \\
(\mathrm{mmol} / \\
\mathrm{mol}^{\mathrm{a}}\end{array}$ & $\mathrm{HbA}_{1 \mathrm{c}}(\%)^{\mathrm{a}}$ & Ketoacidosis \\
\hline I & 25 & 67 & Background retinopathy & & 66 & 8.2 & \\
\hline II & 27 & 55 & & & 40 & 5.8 & \\
\hline $\mathrm{III}^{\mathrm{b}}$ & 15 & 31 & Keratoconus & & 44 & 6.2 & + \\
\hline IV & 1 & 25 & & & 54 & 7.1 & \\
\hline $\mathrm{V}$ & 24 & 49 & Retinopathy & Peripheral vascular disease & 65 & 8.1 & + \\
\hline VI & 18 & 44 & $\begin{array}{l}\text { Optic atrophy } \\
\text { Background retinopathy }\end{array}$ & Neuropathy & 51 & 6.8 & \\
\hline $\mathrm{VII}^{\mathrm{b}}$ & 18 & 58 & Proliferative retinopathy & $\begin{array}{l}\text { Neurogenic bladder } \\
\text { Neuropathy }\end{array}$ & 57 & 7.4 & + \\
\hline VIII & 14 & 27 & & & 60 & 7.6 & \\
\hline
\end{tabular}

${ }^{a}$ Average over four measurements

${ }^{\mathrm{b}}$ Positive for islet autoantibodies

'+' indicates presence of ketoacidosis 
variant was not in linkage disequilibrium $\left(r^{2}<0.01\right)$ with other coding variants in the WFS1 gene. To confirm that the association signal observed at the p.R558C variant is independent of the common variant association, we performed association analysis using a coding variant (p.R611H, rs734312) that shows statistically significant association with type 2 diabetes (OR 1.07, $p=1.29 \times 10^{-10}$ ) in recent large-scale exome-wide association studies [19]. This coding variant is also in strong linkage disequilibrium $\left(r^{2}=0.716\right)$ with a common variant, rs10010131, which has previously been associated with type 2 diabetes [31]. In a joint analysis of the two variants (p.R558C and p.R611H) for association with type 2 diabetes, the strength of association for the p.R558C variant was essentially unchanged (OR 1.59, $p=0.0344$ in the HUGR casecontrol dataset and OR 4.34, $p=0.02$ in the T2D-GENES dataset). The association signal was also unaffected by the inclusion of covariates such as age and sex (data not shown). This demonstrated that the p.R558C variant is associated with an increased risk of type 2 diabetes independent of the common variant association at this locus.

Haplotype analysis of the p.R558C variant To determine the haplotype of origin for the p.R558C variant, we estimated haplotypes spanning coding variants in the WFS1 gene using exome data for individuals in the Ashkenazi Jewish T2DGENES dataset [19]. Haplotype reconstruction using the statistical method PHASE [23] (v2.1; http://stephenslab. uchicago.edu/phase/download.html; accessed 1 November 2017) identified seven haplotypes with an estimated frequency greater than $1 \%$. Of these, a single haplotype with frequency $1.2 \%$ was estimated to be present in all 21 individuals with the minor allele ' $\mathrm{T}$ ' at the p.R558C variant (Fig. 1). This was the same haplotype that was observed in targeted sequence data for the one homozygous individual from the recent sequencing study [15]. Furthermore, analysis of sequence reads from p.R558C heterozygotes in the T2DGENES Ashkenazi Jewish data showed that the risk allele (T) at the p.R558C variant is located on the same haplotype as the risk allele (A) for the p.R611H common variant. Therefore, the p.R558C allele likely arose on a single haplotype background and represents a founder mutation in the Ashkenazi Jewish population.

In silico analysis of the $\mathrm{p} . \mathrm{R} 558 \mathrm{C}$ variant The $\mathrm{p} . \mathrm{R} 558 \mathrm{C}$ mutation is located in exon 8 of the WFS1 gene and changes a hydrophilic, charged amino acid (arginine) to a neutral one (cysteine). The protein encoded by WFS1, wolframin, has nine predicted transmembrane domains and the 558 amino acid is located in a predicted extracellular loop (Fig. 2a). The amino acid substitution is predicted to be highly deleterious and damaging to protein structure by multiple in silico tools for assessing the impact of amino acid substitutions on protein structure and function, including PolyPhen-2 (probably damaging), SIFT (deleterious), MutationTaster (disease causing) and CADD (score $=30$ ) [34]. The high Grantham score for this variant (180 for an arginine to cysteine substitution) also indicates a deleterious change. Multiple sequence alignment across species showed that the amino acid at position 558 is conserved from humans to zebrafish to fruit flies (Fig. 2b). Another variant that affects the same amino acid residue (NM_001145853:c.1673G>A; p.R558H) has also been reported in multiple individuals with Wolfram syndrome from France $[35,36]$ and has been predicted to be deleterious by multiple in silico tools. Therefore, multiple lines of evidence indicate that the amino acid residue at position 558 is functionally important for the protein encoded by WFSI.
Fig. 1 Haplotype analysis of the p.R558C variant in the Ashkenazi Jewish population. Haplotype frequencies for ten coding single nucleotide variants in exon 8 of the WFS1 gene were estimated using exome sequence data from 858 Ashkenazi Jewish individuals. The haplotype with the minor allele ' $\mathrm{T}$ ' at the p.R558C variant was observed to have an allele frequency of $1.2 \%$. This haplotype was identical to the haplotype observed in a European individual homozygous for the p.R558C variant

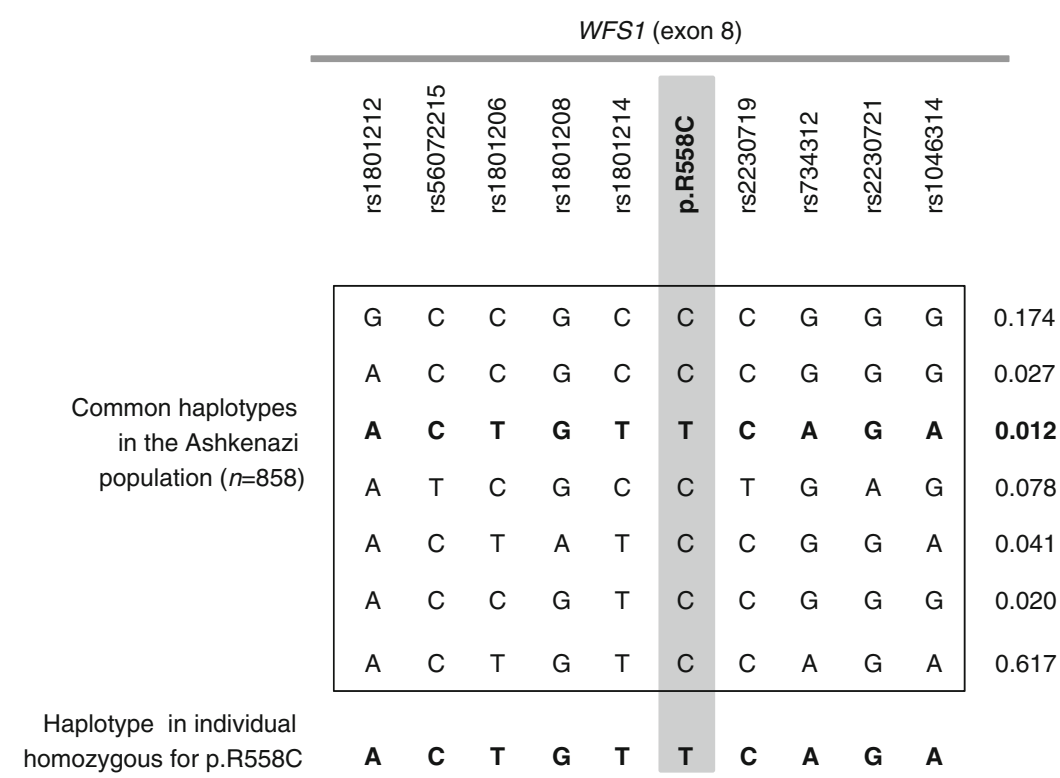


Fig. 2 Location of the p.R558C variant in WFS1 protein and conservation across species. (a) Predicted structure of wolframin protein (adapted from Tranebjærg et al [41] (1993-2018, University of Washington, Seattle, WA, USA) with the locations of the nine putative transmembrane domains. The p.R558C variant is located in an extracellular loop. (b) Multiple sequence alignment of amino acids in the neighbourhood of the 558 position across various species. Arginine is strongly conserved across species including mammals and vertebrates. Chimp, chimpanzee; $\mathrm{ER}$, endoplasmic reticulum

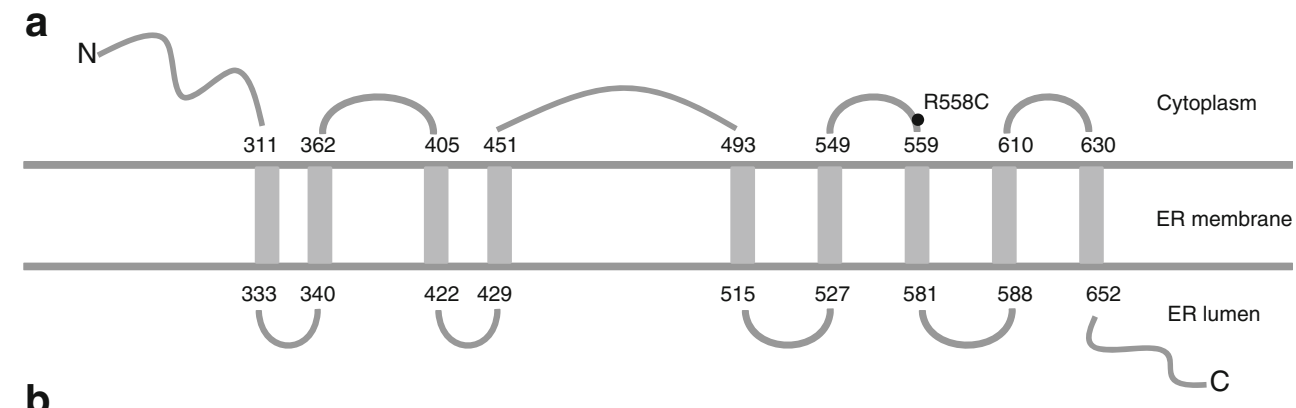

b

$\begin{array}{ll}\text { Human } & 537 \\ \text { Chimp } & 493 \\ \text { Macaque } & 537 \\ \text { Cow } & 536 \\ \text { Mouse } & 536 \\ \text { Rat } & 539 \\ \text { Chicken } & 522 \\ \text { Zebrafish } & 534 \\ \text { Fruit fly } & 483 \\ \text { Frog } & 511\end{array}$

CFMWCELSVVILLESTGLGLLR ASIGYFLFLFALPILVAGLALVGVLQFA CFMWCELSVVILLESTGLGLLR ASIGYFLFLFALPILVAGLALVGVLQFA CFMWCELSVVILLESTGLGLLR ASIGYFLFLFALPILVAGLALVGVLQFA CFMWCELSVVILLESTGLGLVR ASVGYFLFLFALPILVFALALMGVVQLA CFMWCELSVVILLQSTGLGLVR ASIGYFLFLFALPILVAGLALMGTVQFA CFMWCELSVVILLQSTGLGLVR ASIGYFLFLFALPILVAGLALMGTVQFA CFMWCELSVVILRESSGIGLVR ASIGYFLFLFALPVLGVGIALMCLVHFI CFIWCEVCVTLLQESTVLGLMR SVVGYLLFFFALPVLSLALAAVMLVQLV TLSWLQVCIATSQSSTVFGVMR AALGLAGIVLFLPLFGIVALLVPVFVAI CFMWCELSVVIIRESTTIGLIRASVGYFLFLFALPILIVGIVVMCIVHFI

\section{Discussion}

In this paper, we describe a missense mutation in the WFS1 gene that is a low-frequency variant (minor allele frequency $1.4 \%$ ) in the Ashkenazi Jewish population and causes a mild form of Wolfram syndrome. Among 475 individuals of Ashkenazi Jewish ancestry with type 1 diabetes, we identified eight homozygotes for this p.R558C variant. Although a large number of pathogenic mutations have been identified in individuals with Wolfram syndrome, very few mutations have been detected in multiple unrelated individuals. A single insertion mutation was reported to be frequent in Spanish Wolfram syndrome pedigrees in 2001 [37] but there have been no other reports of any founder mutations for Wolfram syndrome. This variant is a bona fide founder mutation that is likely to be a frequent cause of non-autoimmune young-onset diabetes in Ashkenazi Jewish individuals (expected incidence of 1 in every 5100 births).

The Wolfram syndrome phenotype is less severe in p.R558C homozygous individuals than in those with classical Wolfram syndrome, with a later age of onset of diabetes and an absence of optic atrophy in the majority of homozygotes. It is, therefore, less likely to be diagnosed clinically. Some individuals with Wolfram syndrome are misdiagnosed as having type 1 diabetes in the absence of optic atrophy [13, 14]. A diagnosis of Wolfram syndrome is important since it can allow for a comprehensive clinical evaluation for phenotypes associated with Wolfram syndrome, clinical monitoring and management of auditory and ocular phenotypes. Early diagnosis of individuals who are homozygous for p.R558C can facilitate better management of the disease and its phenotypes [27]. Therefore, genetic testing for this variant should be considered in individuals of Ashkenazi Jewish ancestry diagnosed with non-autoimmune early-onset diabetes.

We found that two of the eight homozygotes with type 1 diabetes had additional Wolfram syndrome-relevant phenotypes (optic atrophy and neurogenic bladder, respectively). Many of the phenotypes associated with Wolfram syndrome, such as hearing loss and neurodegeneration, are typically detected after the diagnosis of Wolfram syndrome and manifest over time. A previously reported homozygote for this variant [16], was diagnosed with optic atrophy at the age of 53 years. Three of the eight homozygous individuals identified in our study were less than 35 years old at recruitment and the possibility that they would develop other Wolfram syndrome-related phenotypes later in life cannot be ruled out. Moreover, the individuals with type 1 diabetes were not specifically evaluated for phenotypes such as hearing loss or neurodegeneration and were not available for follow-up phenotyping. Further studies in Ashkenazi Jewish or other populations are needed to replicate our findings and determine the penetrance of this variant for diabetes and other phenotypes observed in Wolfram syndrome. We also observed that two of the eight homozygotes were positive for islet-cell autoantibodies. This was consistent with results from a previous study of 50 individuals with Wolfram syndrome in which $10 \%$ of the individuals with Wolfram syndrome were positive for autoantibodies [28] compared with $86 \%$ of individuals with type 1 diabetes. This suggests that the presence of autoantibodies should not be used to exclude the possibility of Wolfram syndrome in individuals diagnosed with young-onset diabetes. 
Using genotype and sequence data from two Ashkenazi Jewish case-control cohorts for type 2 diabetes, we demonstrated that the p.R558C variant is associated with an increased risk of diabetes in heterozygous carriers. Although common variants at the WFS1 gene locus have been associated with an increased risk of type 2 diabetes, heterozygous loss-of-function mutations in WFS1 did not show an association with risk for type 2 diabetes (ESM Table 3). The p.R558C variant has a more severe phenotype in heterozygous individuals and a less severe phenotype in homozygous individuals compared with loss-of-function variants. Virtually all of the mutations in WFS1 that have been associated with autosomal dominant phenotypes, such as diabetes [6], congenital cataract [8], sensorineural hearing loss [5] and optic atrophy (without diabetes), correspond to missense mutations. Unlike loss-offunction variants, missense mutations can have a dominant negative effect on protein function [38] and this may explain the association of the p.R558C variant with risk for type 2 diabetes in heterozygotes.

Most of the mutations in WFS1 that have been reported in the literature are based on sequencing of individuals that have been clinically diagnosed with Wolfram syndrome. As a result, there is likely to be an ascertainment bias against mutations that lead to less severe forms of the syndrome. Indeed, we first identified this variant in a targeted sequencing study of individuals with nonautoimmune diabetes [15]. Our study highlights the potential of large-scale DNA sequencing studies to identify mutations that cause less severe forms of Mendelian disease. It is likely that similar mutations remain to be discovered for other recessive disorders.

A number of recessive disease-causing mutations that have high frequency in the Ashkenazi Jewish population have been identified over several decades [39]. Carrier screening is recommended for many of these recessive diseases in Ashkenazi Jewish individuals [40]. The p.R558C variant has an estimated carrier frequency of 1/36 in Ashkenazi Jewish individuals, similar to that of disorders such as Tay-Sachs disease [39]. Therefore, this variant should be added to panels of genetic variants that are routinely screened for in individuals of Ashkenazi Jewish descent.

In conclusion, the p.R558C variant represents a frequent cause of a mild form of Wolfram syndrome in individuals of Ashkenazi Jewish ancestry that is difficult to diagnose clinically. Genetic testing for this variant in individuals diagnosed with early-onset diabetes and who are negative for autoimmune antibodies can identify individuals with undiagnosed Wolfram syndrome. Timely diagnosis of Wolfram syndrome can improve an individual's prognosis, detect Wolfram syndrome-related complications and enable genetic counselling of family members. Our results add to the complexity of genotype-phenotype relationships between mutations in WFS1 and human disease.
Acknowledgements The Ashkenazi Jewish T2D-GENES data used in this study was obtained from the $\mathrm{NIH}$ dbGaP repository (study accession phs001095.v1.p1) and supplied by the Albert Einstein College of Medicine and Broad Institute. The Ashkenazi T2D-GENES Sequencing study was conducted by the Ashkenazi T2D-GENES Sequencing study investigators and supported by the National Institute of Diabetes and Digestive and Kidney Diseases (NIDDK). We thank R. Blagieva, S. Claudi-Boehm, B. Manfras and W. Kratzer (University of Ulm, Germany) for phenotyping.

Data availability The genotype data generated in this study is available from the corresponding author on reasonable request.

Funding This project was supported by start-up funds from the Department of Pediatrics at the University of California San Diego and a gift from Rady Children's Hospital, San Diego to VB. BOB is supported by the Lee Kong Chian School of Medicine, Nanyang Technological University Start Up Grant MOE AcRF Tier 1 (2015-T1-001-258), NTU-NHG Metabolic Diseases Collaboration Grant (MDCG/15006), Deutsche Forschungsgemeinschaft (DFG, GrK 1041) and State BadenWuerttemberg, Germany.

Duality of interest The authors declare that there is no duality of interest associated with this manuscript.

Contribution statement VB conceived the project, obtained sequence and genotype data, analysed and interpreted the data and wrote the manuscript. BOB acquired phenotype data and reviewed and edited the manuscript. $\mathrm{AD}$ acquired genotype and phenotype data, contributed to interpretation of the data and reviewed and edited the manuscript. All authors approved the final version of the manuscript. VB is the guarantor of this work, had full access to all the data in the study and takes responsibility for the integrity of the data and accuracy of the data analysis.

\section{References}

1. Barrett TG, Bundey SE, Macleod AF (1995) Neurodegeneration and diabetes: UK nationwide study of Wolfram (DIDMOAD) syndrome. Lancet 346:1458-1463

2. Inoue H, Tanizawa Y, Wasson J et al (1998) A gene encoding a transmembrane protein is mutated in patients with diabetes mellitus and optic atrophy (Wolfram syndrome). Nat Genet 20:143-148

3. Fonseca SG, Fukuma M, Lipson KL et al (2005) WFS1 is a novel component of the unfolded protein response and maintains homeostasis of the endoplasmic reticulum in pancreatic $\beta$-cells. J Biol Chem 280:39609-39615

4. Young TL, Ives E, Lynch E et al (2001) Non-syndromic progressive hearing loss DFNA38 is caused by heterozygous missense mutation in the Wolfram syndrome gene WFS1. Hum Mol Genet 10:2509-2514

5. Bespalova IN, Van Camp G, Bom SJ et al (2001) Mutations in the Wolfram syndrome 1 gene (WFS1) are a common cause of low frequency sensorineural hearing loss. Hum Mol Genet 10:2501-2508

6. Bonnycastle LL, Chines PS, Hara T et al (2013) Autosomal dominant diabetes arising from a Wolfram syndrome 1 mutation. Diabetes 62:3943-3950

7. Rendtorff ND, Lodahl M, Boulahbel $\mathrm{H}$ et al (2011) Identification of p.A684V missense mutation in the WFS1 gene as a frequent cause of autosomal dominant optic atrophy and hearing impairment. Am J Med Genet A 155A:1298-1313

8. Berry V, Gregory-Evans C, Emmett W et al (2013) Wolfram gene (WFS1) mutation causes autosomal dominant congenital nuclear cataract in humans. Eur J Hum Genet 21:1356-1360

9. De Franco E, Flanagan SE, Yagi T et al (2017) Dominant ER stressinducing WFS1 mutations underlie a genetic syndrome of neonatal/ 
infancy onset diabetes, congenital sensorineural deafness and congenital cataracts. Diabetes 66:2044-2053

10. Cano A, Rouzier C, Monnot S et al (2007) Identification of novel mutations in WFS1 and genotype-phenotype correlation in Wolfram syndrome. Am J Med Genet A 143A:1605-1612

11. Astuti D, Sabir A, Fulton P et al (2017) Monogenic diabetes syndromes: locus-specific databases for Alstrom, Wolfram, and Thiamine-responsive megaloblastic anemia. Hum Mutat 7:764-777

12. Chaussenot A, Bannwarth S, Rouzier C et al (2011) Neurologic features and genotype-phenotype correlation in Wolfram syndrome. Ann Neurol 69:501-508

13. Blanco-Aguirre ME, la Parra DR, Tapia-Garcia H et al (2015) Identification of unsuspected Wolfram syndrome cases through clinical assessment and WFS1 gene screening in type 1 diabetes mellitus patients. Gene 566:63-67

14. Zmyslowska A, Borowiec M, Fichna P et al (2014) Delayed recognition of Wolfram syndrome frequently misdiagnosed as type 1 diabetes with early chronic complications. Exp Clin Endocrinol Diabetes 22:35-38

15. Bansal V, Gassenhuber J, Phillips T et al (2017) Spectrum of mutations in monogenic diabetes genes identified from high-throughput DNA sequencing of 6,888 individuals. BMC Med 15:213

16. Lieber DS, Vafai SB, Horton LC et al (2012) Atypical case of Wolfram syndrome revealed through targeted exome sequencing in a patient with suspected mitochondrial disease. BMC Med Genet 13:3

17. Orban T, Sosenko JM, Cuthbertson D et al (2009) Pancreatic islet autoantibodies as predictors of type 1 diabetes in the Diabetes Prevention Trial-Type 1. Diabetes Care 32:2269-2274

18. Bronstein M, Pisanté A, Yakir B, Darvasi A (2008) Type 2 diabetes susceptibility loci in the Ashkenazi Jewish population. Hum Genet 124:101-104

19. Fuchsberger C, Flannick J, Teslovich TM et al (2016) The genetic architecture of type 2 diabetes. Nature 536:41-47

20. He C, Holme J, Anthony J (2014) SNP genotyping: the KASP assay. Methods Mol Biol 1145:75-86

21. Willer CJ, Li Y, Abecasis GR (2010) METAL: fast and efficient meta-analysis of genomewide association scans. Bioinformatics 26: 2190-2191

22. Lim ET, Liu YP, Chan Y et al (2014) A novel test for recessive contributions to complex diseases implicates Bardet-Biedl syndrome gene BBS10 in idiopathic type 2 diabetes and obesity. Am J Hum Genet 95:509-520

23. Stephens M, Smith NJ, Donnelly P (2001) A new statistical method for haplotype reconstruction from population data. Am J Hum Genet 68:978-989

24. Bansal V, Bafna V (2008) HapCUT: an efficient and accurate algorithm for the haplotype assembly problem. Bioinformatics 24:i153i159
25. Lek M, Karczewski KJ, Minikel EV et al (2016) Analysis of protein-coding genetic variation in 60,706 humans. Nature 536 : 285-291

26. NHLBI Exome Sequencing Project (2012) GO Exome Sequencing Project (ESP). Available at http://evs.gs.washington.edu/EVS/. Accessed 1 Sept 2017

27. Urano F (2016) Wolfram syndrome: diagnosis, management, and treatment. Curr Diab Rep 16:6

28. Rohayem J, Ehlers C, Wiedemann B et al (2011) Diabetes and neurodegeneration in Wolfram syndrome: a multicenter study of phenotype and genotype. Diabetes Care 34:1503-1510

29. Kinsley BT, Swift M, Dumont RH, Swift RG (1995) Morbidity and mortality in the Wolfram syndrome. Diabetes Care 18:1566-1570

30. Barrett TG, Bundey SE (1997) Wolfram (DIDMOAD) syndrome. J Med Genet 34:838-841

31. Sandhu MS, Weedon MN, Fawcett KA et al (2007) Common variants in WFS1 confer risk of type 2 diabetes. Nat Genet 39:951-953

32. Fawcett KA, Wheeler E, Morris AP et al (2010) Detailed investigation of the role of common and low-frequency WFS1 variants in type 2 diabetes risk. Diabetes 59:741-746

33. Kawamoto T, Horikawa Y, Tanaka T, Kabe N, Takeda J, Mikuni M (2004) Genetic variations in the WFS1 gene in Japanese with type 2 diabetes and bipolar disorder. Mol Genet Metab 82:238-245

34. Kircher M, Witten DM, Jain P, O Roak BJ, Cooper GM, Shendure J (2014) A general framework for estimating the relative pathogenicity of human genetic variants. Nat Genet $46: 310-315$

35. Colosimo A, Guida V, Rigoli L et al (2003) Molecular detection of novel WFS1 mutations in patients with Wolfram syndrome by a DHPLC-based assay. Hum Mutat 21:622-629

36. Smith CJA, Crock PA, King BR, Meldrum CJ, Scott RJ (2004) Phenotype-genotype correlations in a series of Wolfram syndrome families. Diabetes Care 27:2003-2009

37. Gómez-Zaera M, Strom TM, Rodríguez B, Estivill X, Meitinger T, Nunes V (2001) Presence of a major WFS1 mutation in Spanish Wolfram syndrome pedigrees. Mol Genet Metab 72:72-81

38. Cryns K, Pfister M, Pennings RJE et al (2002) Mutations in the WFS1 gene that cause low-frequency sensorineural hearing loss are small non-inactivating mutations. Hum Genet 110:389-394

39. Strom CM, Crossley B, Redman JB et al (2004) Molecular screening for diseases frequent in Ashkenazi Jews: lessons learned from more than 100,000 tests performed in a commercial laboratory. Genet Med 6:145-152

40. Gross SJ, Pletcher BA, Monaghan KG, Practice P, Committee G (2008) Carrier screening in individuals of Ashkenazi Jewish descent. Genet Med 10:54-56

41. Tranebjærg L, Barrett T, Rendtorff ND (2009) WFS1-related disorders [updated 19 Dec 2013]. In: Adam MP, Ardinger HH, Pagon RA et al (eds) GeneReviews ${ }^{\circledR}$ [Internet]. University of Washington, Seattle 